\title{
Decoupling Architecture for All-to-all Computation
}

\author{
Atsushi Hori \\ RIKEN AICS \\ ahori@riken.jp \\ Yasumasa Joti \\ JASRI/RIKEN SPring-8 Center \\ joti@spring8.or.jp \\ Mitsuhiro Yamaga \\ JASRI/RIKEN SPring-8 Center \\ myamaga@spring8.or.jp \\ Yuji Sugita \\ RIKEN AICS \\ sugita@riken.jp
}

\author{
Kazumi Yoshinaga \\ RIKEN AICS \\ kazumi.yoshinaga@riken.jp \\ Kensuke Okada \\ JASRI/RIKEN SPring-8 Center \\ k.okada@spring8.or.jp \\ Takaki Hatsui \\ RIKEN SPring-8 Center \\ hatsui@spring8.or.jp \\ Yutaka Ishikawa \\ Univ. of Tokyo/RIKEN AICS \\ yutaka.ishikawa@riken.jp
}

\author{
Atsushi Tokuhisa \\ RIKEN AICS \\ tokuhisa@riken.jp \\ Takashi Sugimoto \\ JASRI/RIKEN SPring-8 Center \\ takashi.sugimoto@spring8.or.jp \\ Makina Yabashi \\ RIKEN SPring-8 Center \\ yabashi@spring8.or.jp \\ Nobuhiro Go \\ JAEA \\ go.nobuhiro@jaea.go.jp
}

\begin{abstract}
We propose a new software framework, named "decoupling architecture", for all-to-all computation. In this framework, the user's kernel code and well-tuned parallel control code are clearly separated to allows users to move smoothly from running sequential programs on a single-node server to fully utilizing a powerful machine, such as the $\mathrm{K}$ computer. In an evaluation of our prototype, the proposed decoupling architecture introduced some overhead. However, we show that the overhead can be reduced.
\end{abstract}

\section{Categories and Subject Descriptors}

D.1.3 [PROGRAMMING TECHNIQUES]: Concurrent Programming-Parallel programming; D.2.11 [SOFTWARE ENGINEERING]: Software Architecture-Domain-specific architectures; D.4.4 [OPERATING SYSTEMS]: Communications Management-Input/output

\section{Keywords}

Big Data, X-Ray Free Electron Laser, MPI

\section{INTRODUCTION}

We succeeded in developing software running on the $\mathrm{K}$ computer [8] to analyze big data obtained by the light source SACLA (X-ray Free Electron Laser) [9]. Soon after, we decided to re-design the software to be more general, so that users who are not familiar with big parallel machines can easily take advantage of the full power of the $\mathrm{K}$ computer. Because so many tasks must be carefully done to fully use

Permission to make digital or hard copies of all or part of this work for personal or classroom use is granted without fee provided that copies are not made or distributed for profit or commercial advantage and that copies bear this notice and the full citation on the first page. Copyrights for components of this work owned by others than ACM must be honored. Abstracting with credit is permitted. To copy otherwise, or republish, to post on servers or to redistribute to lists, requires prior specific permission and/or a fee. Request permissions from permissions@acm.org.

EuroMPI/ASIA'14 September 9-12, 2014, Kyoto, Japan Copyright 2014 ACM 978-1-4503-2875-3/14/09 ...\$15.00.

http://dx.doi.org/10.1145/2642769.2642801 big parallel machines [5], developing a well-tuned parallel program is difficult.

Software frameworks $[6,7]$ are now widely used in software development to achieve higher productivity, not only in the enterprise field, but also in the high-performance computing (HPC) field [3]. A software framework is essentially a software library, regardless of the control direction. A typical framework is written in object-oriented language. Users must write their program with the programming language with which the framework is written and to learn about the class system provided by the framework. IT programmers and engineers may not be concerned with these requirements; however, research scientists and engineers of fields other than the IT field may be reluctant to convert existing programs because their primary interest is not software development. If they have a Fortran program, for example, then they have to rewrite the program with the programming language that the framework can support. This may make those researchers and engineers hesitate to do so, and so they might lose the chance to have a powerful investigating tool to speed up their research.

The decoupling architecture proposed in this paper bridges the gap between users having sequential programs that cannot be used with big parallel computers. Users of the decoupling architecture are free to choose the programming language for their programs. The decoupling architecture has only a few rules, and it does not inherit classes. Thus, it can be a good starting point for scientific researchers and engineers to use big parallel machines.

In the next section, the co-designed program is introduced. In Section 3, our proposed decoupling architecture is explained. Then the prototype of the decoupling architecture is evaluated. Following this section, related work and summary sections are given.

\section{SACLA AND THE K COMPUTER}

To explain our proposed architecture, we use SACLA image data. SACLA is the name of the X-Ray Free Electron Laser facility owned and operated by RIKEN [9]. SACLA can produce a series of intense X-Ray pulses so that the $3 \mathrm{D}$ structures of tiny particles, such as proteins, cells, and 


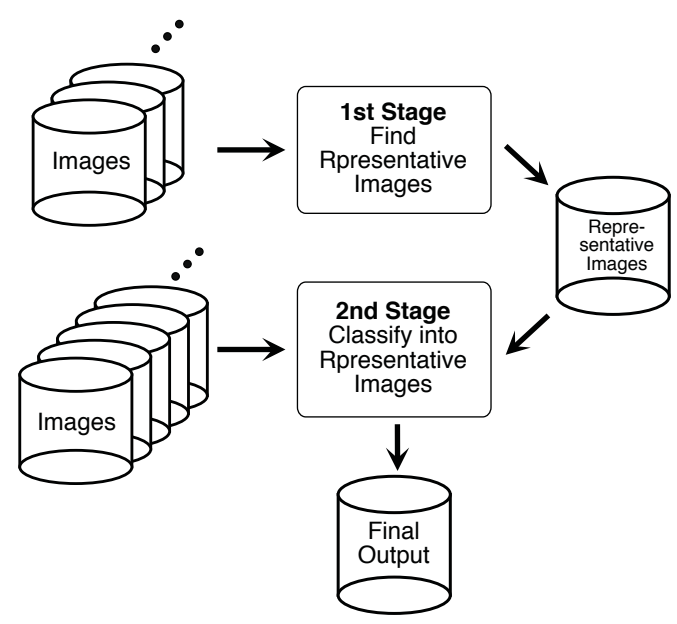

Figure 1: Block Diagram

viruses, can be analyzed. To reduce the quantum noise in images, 100 images must be averaged. The particles to be analyzed are so small that it is difficult to control their orientations, and so the images obtained by SACLA must be categorized by each image orientation. To obtain the 3D structure of a particle, images shot from every possible orientation are needed. Thus, the typical total number of images to be shot is one million to analyze a $3 \mathrm{D}$ structure, depending on the size of the particle (the number of atoms composing the particle) and the target resolution. SACLA can take as many as 30 images per second and one million images in approximately 10 hours. The typical image size is approximately $20 \mathrm{MB}$, and the complete image dataset size is $20 \mathrm{~TB}$.

Tunable experimental parameters are used to obtain good diffraction images. Therefore, the images taken by SACLA must be analyzed in the middle of an experiment, i.e., as soon as possible. If the experiment goes wrong, then the experiment must be aborted and restarted with a different experimental parameter set. To analyze the diffraction images on the fly, a 10-peta-flops $\mathrm{K}$ computer [8] is used. This computer is owned and operated by RIKEN AICS, located 50 miles away from where SACLA is located. We succeeded in transferring the image data from SACLA to the K computer with the highest speed that SACLA can produce [1].

The kernel program to analyze the image data was developed by the SACLA research team [11]. The System Software Research Team at RIKEN AICS parallelized the program and optimized the I/O performance. The entire analysis requires two stages. The first stage finds representative images, each of which represents a certain orientation of the target particle and is differentiated from other orientations based on a certain solid angle. This computation takes place on a subset (approximately 10\%) of all images.

\subsection{First Stage}

Finding the representative images is a classic clustering computation. Thus, all images must be compared with all others. As shown in Figure 2, the matrix represents all-toall computation, and the rows and columns represent image data. Since the comparison of two images is commutative, only the upper (or lower) triangle half of the matrix must

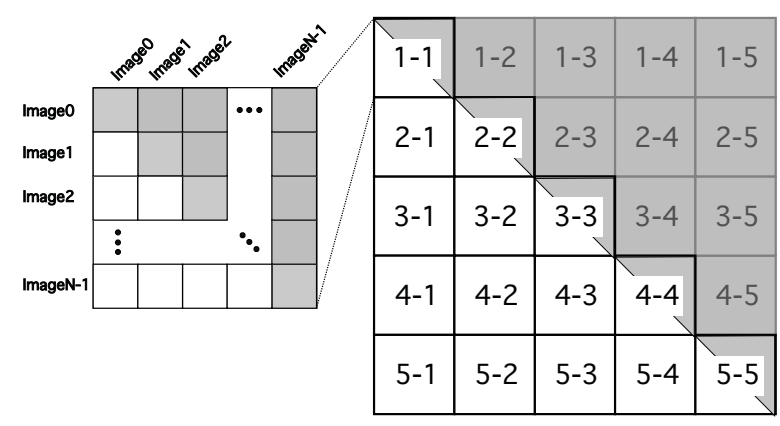

Figure 2: All-to-all Computation

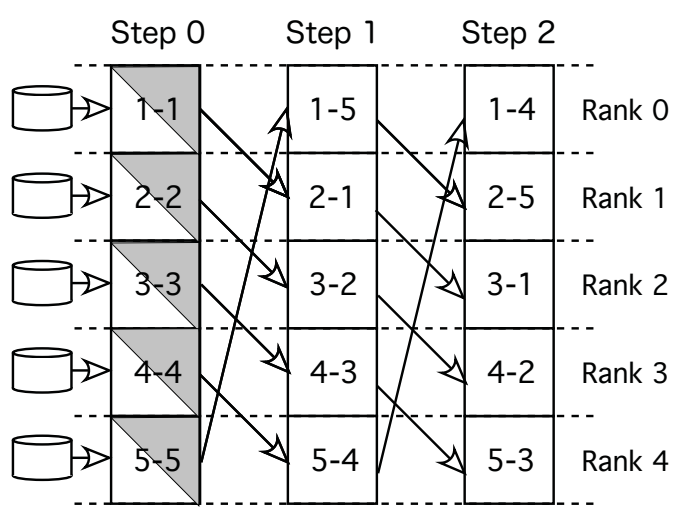

Figure 3: Load Balancing

be computed.

A naive implementation of this algorithm can easily result in 1) load imbalance, and 2) frequent file read operations. For example, we assume that the required computation of each row of the matrix shown on the right in Figure 2 is assigned to an MPI process that does the clustering. Because the matrix has 5 rows, 5 MPI processes are allocated. The MPI process assigned to the uppermost row, denoted as 1-1 to 1-5, analyzes data files $\mathbf{1}$ to $\mathbf{5}$. Here, each file contains data of a certain number of images. The lowermost row, however, has only one file $\mathbf{5}$ for the clustering. Thus, the MPI process assigned to the uppermost column is the busiest, while the node assigned to the lowermost column is the idlest.

From the viewpoint of I/O, the uppermost MPI process must read all 5 files, $\mathbf{1}$ to $\mathbf{5}$, while the lowermost MPI process must only read one file, $\mathbf{5}$. In total, 15 files must be read in this naive implementation, whereas the number of files is only $\mathbf{5}$. When the number of nodes and the number of files increases, the file system is overwhelmed by the number of file read requests.

To solve the two problems of computation load imbalance and too many I/O read requests, we developed the parallel computation scheme shown in Figure 3. The scheme has 3 steps for completing the whole computation when the number of MPI ranks is 5. In the first step, all MPI processes read the file allocated to each process and processes the read data. The next step is started by sending the read data to its neighbor process. The received data and the data read in the first step are then processed. The final step is the 


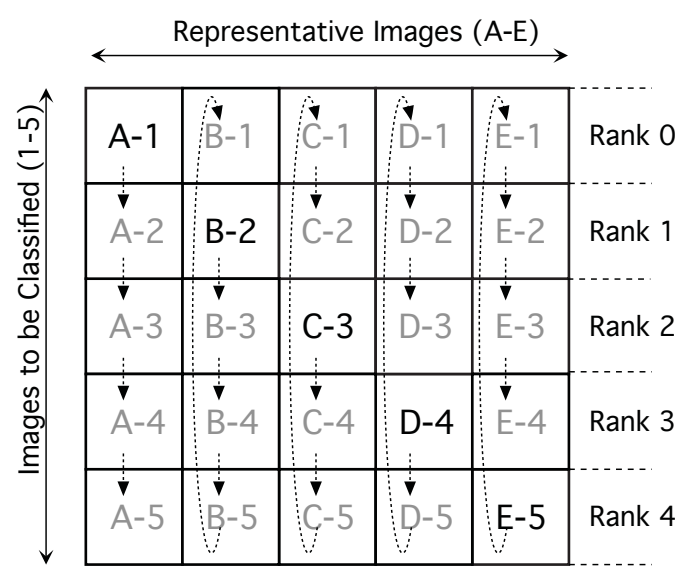

Figure 4: Second Stage

same as the second step (sending the read data). In this way, files are read by MPI processes only once, and the load is completely balanced between MPI processes. The number of steps depends on the number of nodes. The formula below represents the relationship between the number of steps $\left(N_{\text {steps }}\right)$ and the number of MPI processes $(P)$.

$$
N_{\text {steps }}=\lceil(P+1) / 2\rceil
$$

According to this formula, a load imbalance can occur when the number of MPI processes is even. However, this load imbalance is negligible when the number of nodes is large.

\subsection{Second Stage}

In the way described in the previous subsection, representative images can be found. The second stage classifies the remaining images (as mentioned above, 90\% of the images) into the representative images, so that those classified images are averaged to reduce the quantum noise. This process is implemented in a way somewhat similar to that of the first stage. Figure 4 shows an example of this procedure. At the first step, each MPI process reads the assigned representative images and the images to be classified are read. For example, the rank- 0 process in this figure reads the representative image set $\mathbf{A}$ and the image set to be classified $\mathbf{1}$, the rank-1 process reads $\mathbf{B}$ and $\mathbf{2}$, and so on. When the classification is finished, the rank-0 process, for example, sends the representative image data $\mathbf{A}$ to its neighbor, rank-1, receives the other representative image data $\mathbf{E}$ from rank-4, and classifies the image data $\mathbf{1}$ into $\mathbf{B}$. This procedure is repeated 5 times to classify all images.

\subsection{Burst I/O Problem}

In stage 1 and stage 2 above, all input data are read at the beginning of program execution. This bursty I/O pattern may overload the file system. Our target machine, the $\mathrm{K}$ computer, has two types of local file systems. Here, "local" means that the files in a local file system can be accessed inside a job. One is a parallel file system where any file can be accessed from any MPI processes. Another local file system is not a parallel file system and only the associated MPI process can access the files in the file system. The latter file system is named Rank Local File System. The

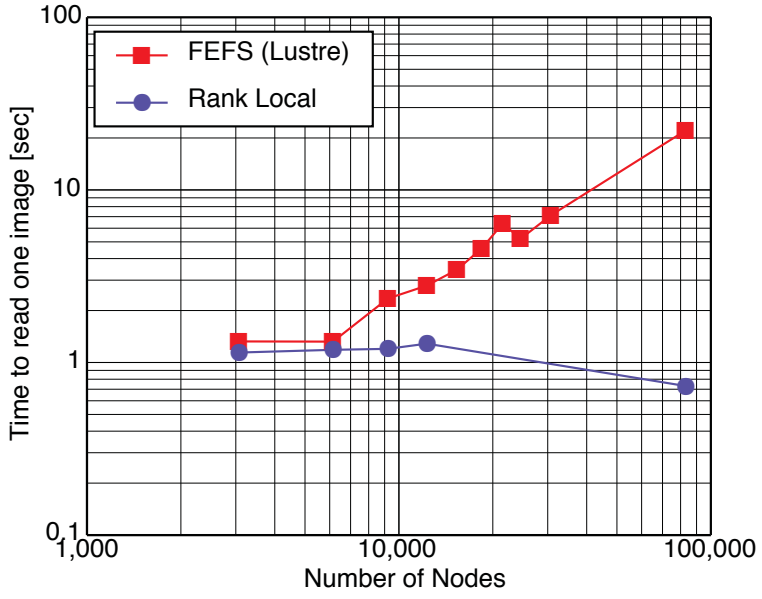

Figure 5: Scalability of File Read

$\mathrm{K}$ computer has another global file system and the files to be accessed in a parallel job are copied into this global file system prior to its execution. The output files of the parallel job are copied when the job terminates. The global parallel file system and the local parallel file system are extensions of the Lustre parallel file system named FEFS[10].

Figure 5 shows the times to read one image data (20 MB) from the local parallel file system (FEFS) and from the Rank Local File System over the number of compute nodes. As shown in this figure, the number of times of using the Rank Local File System is almost independent of the number of nodes, whereas the number of times of using FEFS heavily depends on the number of nodes when the number of nodes gets larger. Thus, the bursty I/O problem can be avoided by using the Rank Local File System.

\section{DECOUPLING ARCHITECTURE}

After developing the above software, we realized that allto-all computation, where any possible combination of two records in a dataset must be processed, can also be used to analyze gene sequencing data, images obtained by electron microscopes, and so on. Therefore, we decided to decouple the kernel code and the parallelization part of the above program. We named the decoupled program $p$ Carp.

We assume that the users of $p$ Carp are familiar with sequential programming, but are not familiar with parallel programming. With the decoupling architecture, $p$ Carp users are required to write sequential programs. All parallelizing tasks are done by $p$ Carp. Note that users may execute the OpenMP thread parallel program if they so desire. In this case, only one $p$ Carp MPI process is invoked in a compute node. We believe this decoupling architecture gives users a clear view of the isolation of the kernel code and the internode parallelization code. All techniques described in Section 2 are programmed in $p$ Carp, which frees users from dealing with parallel programming problems.

We also developed a program named $s$ Carp, which is a sequential program that runs on a single node or a user's laptop PC. Therefore, users can easily develop and debug their kernel codes on familiar platforms. Once their kernel programs are run with $s$ Carp, the codes are guaranteed to run with $p$ Carp on massively parallel machines. 


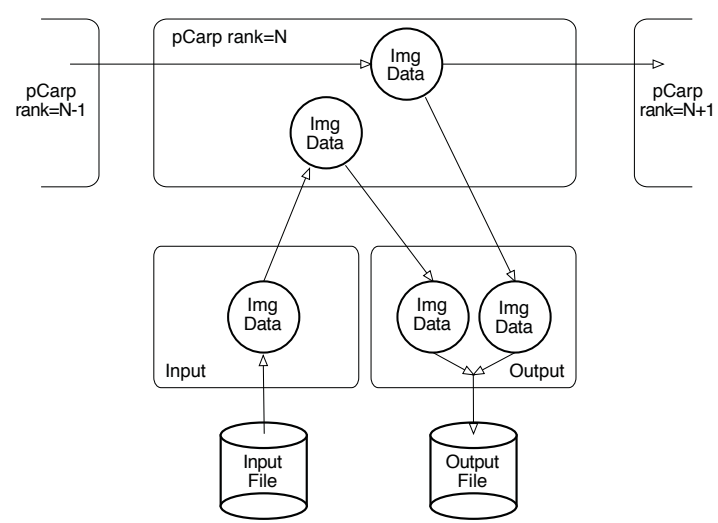

Figure 6: Decoupling Architecture

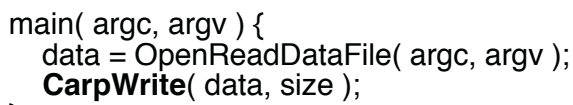

(a) Input Code

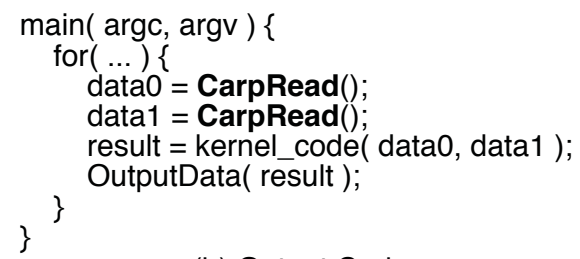

\section{(b) Output Code}

Figure 7: Pseudo code for the input and output processes

Figure 6 shows the decoupled architecture for the all-to-all computation. Each MPI process creates two sub-processes (fork and exec), one to read a file and the other to process two data records and output its result to a file. The data read by the created sub-process are sent back to the parent MPI process $(p$ Carp $)$ and the data are sent to the neighbor node in the way described in Section 2.

The prototype of the $p$ Carp program takes several arguments. Some arguments are to switch stages to 1 or 2 , described in Section 2, followed by some options, such as the program name to read a file (lower left rectangle "Input" in Figure 6) followed by some options, and the program name to classify the data and output the result to a file (lower right rectangle "Output" in Figure 6) followed by some options.

Figure 7 shows the code skeletons of the input and output processes in Figure 6 . the function having bold face names (CarpWrite() and CarpRead()) are the function provided by the $p$ Carp decoupling system. In the upper code

\$ mpiexec pcarp args ./input_prog args ./output_prog args

Figure 8: Execution command

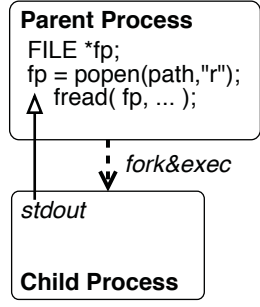

(a) popen( "r" )

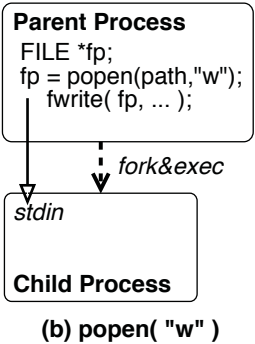

(b) popen( "w" )
Figure 9: popen() function

of Figure 7 shows the code of the input process. In the input program, some arguments (MPI rank number, the number of MPI ranks, etc.) are passed to identify which file to open. The specified file is opened and the read data is passed to the parent $p$ Carp process. In the $p$ Carp process, the read data is preserved in memory and send the data to its neighbor process as shown in Figure 6 to do the all-to-all computation. The input process is created once at the very beginning of the $p$ Carp execution and terminates as soon as the read data is passed to the $p$ Carp process. The lower code is the code of output process where it reads two records (each record data was read by the input process and passed by the $p$ Carp process) and then process those records. Finally its result is output. This procedure is repeated until the all data of the desired all-to-all computation is done. And the output process is create only once at the beginning of the $p$ Carp execution. In those two input and output programs, there is no need of calling the complex MPI functions at all. Thus, users are free from the headache of load balancing and file I/O optimization.

The $p$ Carp MPI program can be invoked in the way shown in Figure 8. It takes the arguments to switch the stage described in Section 2 followed by the arguments of the input program pathname and output program pathname, so that $p$ Carp can invoke them via the popen() libc function.

\section{PROTOTYPE AND EVALUATION}

In our first prototype of $p$ Carp, the popen() libc function is used to create those sub-processes. The popen() function fork () and $\operatorname{exec}()$ a subprocess and a Linux pipe. When the popen ( ) function is called in a read mode, then the pipe connects the standard output of the subprocess and the file pointer of the parent process (Figure [9). When the popen() function is called in a write mode, then the pipe connects the standard input and the file pointer. To exchange data to go back and forth between the MPI process and sub-processes, the pipe create by the popen() function is used. In $p$ Carp, file I/O must be done in the user programs. Consequently, $p$ Carp does not care about the file format.

In the evaluation of this prototype, the execution time is much larger than the original program depending on the data size. For example, when the number of representative images per rank is 8 and the number of images per rank to be analyzed is 10 , the time of stage 2 is 430 seconds with $p$ Carp, while the time of the original program is 240 seconds. According to our investigation, we found that the most of the additional time of $p$ Carp comes from the pipe transmission.

The upper graph in Figure 10 shows the bandwidths of 


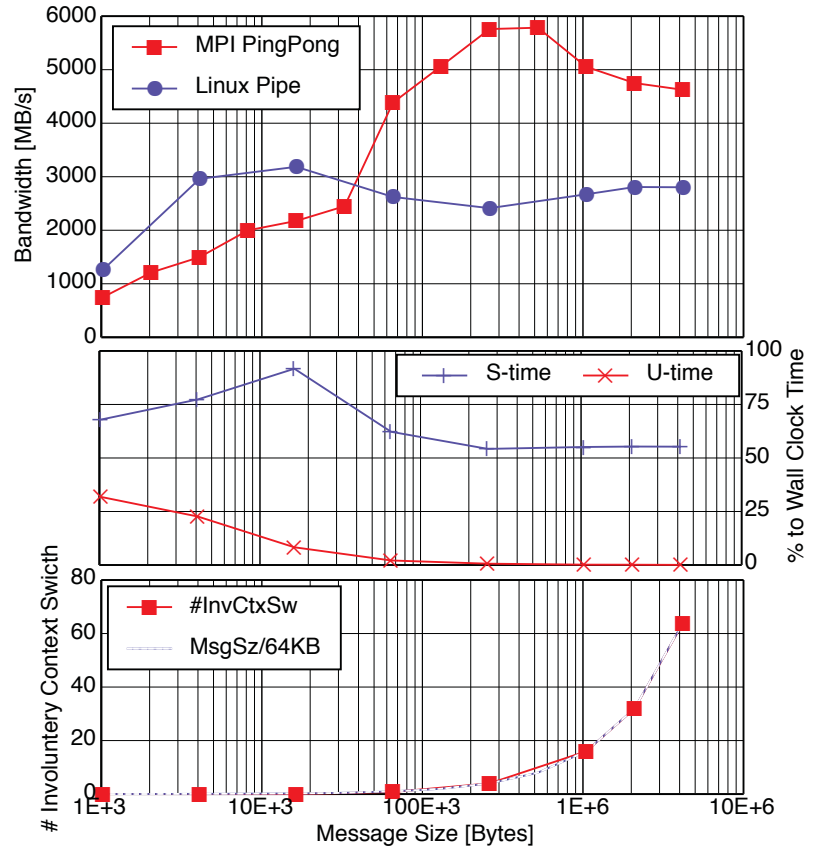

Figure 10: Pipe and MPI Pingpong Bandwidth

the Linux pipe and the intra-node bandwidths of Intel MPI measured by the Intel MPI Benchmark program [2]. Note that we used the Intel Xeon server (E5-2650 v2, $2.60 \mathrm{GHz}$ ), not the $\mathrm{K}$ computer, in this measurement. When the message size is smaller than $64 \mathrm{~KB}$, then the pipe bandwidth is better than that of the MPI, but when the message size is larger than $32 \mathrm{~KB}$, the MPI bandwidth is better than that of the Linux pipe. The typical image size produced by SACLA XFEL is $20 \mathrm{MB}$, and the bandwidth of pipe is not good in comparison with the MPI bandwidth.

The middle graph in Figure 10 shows the percentages of user times (time spent in a user program, U-time) and system times (time spent in an OS kernel, S-time) to the wall clock time. As the message size increases, the time spent in the OS kernel dominates. The sum of the percentage of user time and the percentage of system time is not $100 \%$, because the process is blocked when reading or writing to pipe. When the message size is larger, almost half of its execution time is spent in blocking. The bottom graph shows the number of involuntary context switches that happen when reading or writing to pipe is blocked by the OS kernel.

The size of the pipe buffer inside the Linux kernel is $64 \mathrm{~KB}$. When the pipe buffer is full, the writer process is blocked. When the pipe buffer is empty, the reader process is blocked. The writer process and reader process can run simultaneously when the message size is less than $64 \mathrm{~KB}$, which is the pipe buffer size. When the message size is larger than 64 $\mathrm{KB}$, the write process blocks every time it tries to write the message into pipe. The reader process also blocks because the reader process tries to consume more data than the size of the pipe buffer. Thus, the writer process and reader process perform blocking every time they write to or read from pipe. As shown in the bottom graph in Figure 10, the number of involuntary context switches is proportional to the message size, and the number of context switches is nearly equal to the number of message sizes divided by $64 \mathrm{~KB}^{1}$. Consequently, the writer and reader processes can run only in an alternate manner, with no chance of running simultaneously, even if they are bound to different cores. Thus, only half of the execution time can be utilized, as shown in the middle graph. This situation is observed by the Linux top command. Both reader and write processes can use only $50 \%$ of the CPUs. Thus, in theory, the pipe bandwidth could be doubled.

To improve the pipe problem, a straightforward way is to increase the pipe buffer size in the OS kernel. Unfortunately, its size is hard-coded inside the OS kernel and is therefore difficult to change. The same technique to implement MPI intra-node communication is a possible solution. The MPI bandwidth in Figure 10 is 1.7 times larger than that of pipe. In many MPI implementations, a shared memory segment is used to exchange data between MPI processes. Alternatively, we can develop a Linux device driver having a large enough buffer to hold the entire data exchanged between two processes.

One may argue that users can see the overhead of exchanging data between processes, even when the bandwidth is improved by several times. This depends on the time of kernel code execution. If the kernel code execution time is much larger than that of the inter-process communication, then the overhead is difficult to see. Even when the kernel execution time is not large enough, we believe the easy use of pCarp may still be beneficial for many users.

\section{RELATED WORK}

The proposed decoupling architecture can be thought as a software framework $[6,7]$. In a typical software framework, the user kernel code and the framework code are linked together as a single program. The software framework and the user kernel code are Linux programs in the decoupling architecture.

A well-known map-reduce framework can also be applied to all-to-all computation. KMR is a map-reduce program tuned for the $\mathrm{K}$ computer [4]. In general, map-reduce processing must be repeated for all-to-all computation, and the intermediate result must be saved onto a disk and restored from the disk for later computation. This adds extra overhead, but $p C a r p$ can avoid this situation by shifting data to/from the neighbor MPI processes.

The decoupling architecture has some limitations. One limitation is that the user kernel code must not be an MPI program. The other limitation is that the user kernel code must have two input streams and one output stream.

In spite of those limitations and the pipe overhead described in the previous section, the decoupling architecture can be applied to applications where all-to-all computation takes place.

\section{SUMMARY AND FUTURE WORK}

Through the co-designing of a program to analyze the data obtained by the light source SACLA, we developed the idea of the decoupling architecture. The decoupling architecture is a novel way to implement a software framework. The framework gives users the freedom to choose their own programming language in which to write their kernel codes.

${ }^{1}$ These numbers of context switching are the averages of one million time itereations. 
Therefore, a strongly independent execution environment exists between the framework and the user kernel codes. An evaluation of the decoupling architecture prototype reveals the overhead of exchanging data between the framework and the kernel codes. This overhead is inevitable in the decoupling architecture; however, its ease of use can attract many users.

The current decoupling architecture programs $p$ Carp and sCarp can only handle all-to-all computation. Exploration of other types of computation and a technique to reduce the data exchange overhead are our future work.

\section{ACKNOWLEDGMENTS}

A part of the results in this paper was obtained by using the $\mathrm{K}$ computer at the RIKEN Advanced Institute for Computational Science (Proposal number hp120214).

\section{REFERENCES}

[1] A. Hori, A. Tokuhisa, K. Yoshinaga, T. Kameyama, K. Okada, J. Arai, T. Sugimoto, M. Yamaga, R. Tanaka, Y. Joti, T. Hatsui, M. Yabashi, Y. Sugita, Y. Ishikawa, and N. Go. SACLA and the K Computer. White Paper presented in BDEC Fukuoka, JAPAN, 2014.

[2] Intel Corp. Intel MPI Benchmarks - User Guide and Methodology Description, Revision 3.2.4 edition, 2013.

[3] M. Ioki and S. Chiba. A framework for multiplatform hpc applications. In Proceedings of Programming Models and Applications on Multicores and Manycores, PMAM'14, pages 61:61-61:69, New York, NY, USA, 2007. ACM.

[4] M. Matsuda, N. Maruyama, and S. Takizawa. K mapreduce: A scalable tool for data-processing and search/ensemble applications on large-scale supercomputers. In Cluster Computing (CLUSTER), 2013 IEEE International Conference on, pages 1-8, Sept 2013.

[5] P. H. Mills, L. S. Nyland, J. F. Prins, and J. H. Reif. Software issues in high-performance computing and a framework for the development of hpc applications. Technical report, COMPUTING, U. VISHKIN, ED.: ACM, 1994.

[6] D. Riehle. Framework Design - A Role Modeling Approach. PhD thesis, SWISS FEDERAL INSTITUTE OF TECHNOLOGY ZURICH, 2000.

[7] D. Riehle and T. Gross. Role model based framework design and integration. In Proceedings of the 13th ACM SIGPLAN Conference on Object-oriented Programming, Systems, Languages, and Applications, OOPSLA '98, pages 117-133, New York, NY, USA, 1998. ACM.

[8] RIKEN AICS. The K Computer. http://www.aics.riken.jp/en/.

[9] RIKEN Spring-8 Center. SACLA (XFEL). http://xfel.riken.jp/eng/index.html.

[10] K. Sakai, S. Sumimoto, and M. Kurokawa. High-Performance and Highly Reliable File System for the K computer, July 2012. http://www fujitsu . com/downloads/MAG/vol48-3/paper08.pdf.

[11] A. Tokuhisa, J. Arai, Y. Joti, Y. Ohno, T. Kameyama, K. Yamamoto, M. Hatanaka, B. Gerofi, A. Shimada,
M. Kurokawa, F. Shoji, K. Okada, T. Sugimoto, M. Yamaga, R. Tanaka, M. Yokokawa, A. Hori, Y. Ishikawa, T. Hatsui, and N. Go. High-speed classification of coherent X-ray diffraction patterns on the $\mathrm{K}$ computer for high-resolution single biomolecule imaging. Journal of Synchrotron Radiation, 20(6):899-904, Nov 2013. 